\title{
UN SYSTEME INFERENTIEL ORIENTE OBJET POUR DES APPLICATIONS EN LANGUES NATURELLES
}

\author{
ALAIN BERRENIONNER ${ }^{1}$ - MOUNIA FRED ${ }^{2}{ }^{2}$ - FLAVIO OQUENDO ${ }^{2}$ - JACQUES ROUAULT ${ }^{2}$
}

${ }^{2}$ C.R.I.S.S - Université Pierre Mendès France

B.P. 47 - 38040 Grenoble Cedex 9 FRANCE

Fax : $(+33) .76 .82 .56 .75$

Telex : UNISOG 980910

Tel : $(+33) .76 .82 .54 .15$ et $(+33) 76.82 .54 .06$

E-mails : mounia @icriss.grenet.fr

flavio@criss.grenet.fr

ISéminaire de linguistique française - Université de Fribourg

1700 Fribourg SUISSE

\begin{abstract}
Up to now, there is still no specific model for solving the problem of natural language representation and reasoning. In this paper, we propose an object oriented formalism for supporting knowledge representation, extraction and exploitation in the context of natural language processing.

In the natural language analysis, this system is situated after the morpho-syntax and the linguistic semantics. It represents two classes of concepts: objects of discourse and action schemata, the former resulting from nominal syntagms and the latter from the 'processes'. We are concented bere just by the representation of objects.
\end{abstract}

In the natural language discourse, manipulated objects are complex objects and the reasoning is by nature first inferential and then deductive. To take into account this kind of reasoning we need a suitable representation: a model of inferential objects.

The theoretical foundations of the proposed model are Lesniewski's logical systems: the Calculus of Names and the Mereology. The former is based on a primitive functor called "epsilon" interpreted as $i s-a$, the latter is based on a part-of relation which is called the "ingredience". The whole system is supported by these two primitives and theirs derived functions.

The concepts of our model result from a collaboration between linguists and computer scientists. The main concepts are the intensional and extensional universes, notions and types.

The possible inferential reasoning can be of different types : it can concern the status, the denominations, the structures or the "fonctifs" of the objects.

Key-words : Knowledge Representation, Inferential Reasoning, Object Oriented Modelling, Natural Language Processing, Language Parsing and Understanding.

\section{RESUME}

Dans ce papier, nous proposons un fornalisme orienté objet pour la représentation, l'extraction et l'exploitation des connaissances dans le contexte du traitement des langues naturelles.

Dans un discours en langue naturelle, les objets manipules sont des objets complexes et le raisonnement est avant tout de type inférentiel avant d'être déductif. Pour pouvoir tenir compte de ce type de raisounement, nous avons besoin d'une représentation idoine : un modèle d'objets inférentiels.

Les fondements théoriques de notre modèle sont les systèmes logiques de Lesniewski : le Calcul des Noms at la Mćréologic. Le premier repose sur un foncteur primitif appelé "epsilon" interprété comme est-un, le second sur la relation partie-de appelée "l'ingrédience". Les concepts de notre modele sont le fruit d'une collaboration entre linguistes et informaticiens. Les principaux concepts sont les univers intensionnel et extensionnel, les notions et les types.

Les raisonnements inférentiels possibles sont de différentes sortes : ils peuvent porter sur le statut, les dénominations, les structurels ou les fonctifs.

Mots-clés : Représentation des Connaissances, Raisonnement Inférentiel, Modélisation Orientée Objet, Traitement de la Langue Naturelle, Analyse morphosyntaxique et Compréhension du langage.

\section{1- INTRODUCTION}

Le système présenté ici a pour but la représentation, l'extraction et l'exploitation des connaissances dans le contexte du traitement automatique des langues. On sait [Berrendonner 89] que les raisonnements représentés dans des "discours" en langue naturelle ne sont que rarement déductifs et sont le plus souvent inférenticls. Pour pouvoir tenir compte de ces raisonnements, nous avons besoin d'une représentation idoinc.

Il n'existe pas en effet a l'heure actuelle de modèle spécifique pour résoudre le problème de la représentation 
des connaissances et du raisonnement en langue naturelle. Dans ce document, nous décrivons le formalisme de représentation et certains raisonnements que notre système autorise : c'est un modèle d'objets inférentiels.

Ce modèle est lui-même fondé sur les systèmes logiques de Lesniewski [Lesniewski 89]. Dans ces systèmes, nous utilisons le Calcul des Noms (basé sur la primitive $E$ : "est-un/est-le") et la Méréologie (dont le foncteur de base est "partie-tout", appele ingrédience). En définitive, le modèle objet, et tout le système reposent sur ces deux seules primitives et leurs dérivées.

Dans une chaîne d'analyse du français, ce système se situe apres la morpho-syntaxe ct la sémantique linguistique. Il représente deux familles de concepts : les objets du discours, issus de certains des syntagmes nominaux, et les schémas d'action qui sont issus des procès. Nous ne nous intéressons ici qu'à la representation des objets du discours.

Une première partie est consacrée au modèle à un niveau conceptuel : nous y donnons une présentation générale, suivie des concepts sur lesquels repose notre système ef enfin l'unité de représentation de connaissance choisic.

Les bases logiques permettant ha formalisation, ainsi que des caractéristiques propres au modele sont présentées dans une deuxième section. On donne un exemple de formalisation.

Les troisième et quatrième parties exposent l'organisation des connaissances et les raisonnements possibles sur cette connaissance.

La dernière partie consacrée aux techniques d'implémentation est suivie d'une conclusion.

\section{2 - LE MODELE CONCEPTUEL}

\subsection{Présentation générale}

Notre modèle résulte d'unc collaboration entre linguistes et informaticiens. Il s'appuie sur certains résultats de la psychologie cognitive.

\subsection{Les univers}

Le premicr concept de base du système est celui d'univers. Chaque tmivers est constitué de deux parties : l'intensionnelle I et l'extensionnelle $\mathrm{R}$.

La partie intensionnelle regroupe des "connaissances générales", c'est-à-dire valides dans toutes conditions d'énonciation, donc en fait des objets "logiques" sur lesquels on peut faire des inférences. L'univers extensionnel est un ensemble cohérents d'objets crés par le discours et repérés par des conditions d'énonciation particulières.

Une contradiction entre les objets de l'univers et ceux du discours provoque le passage à un nouvel univers. C'est le cas quand l'évolution du dialogue mène à un conflit (différence de points de vue entre interlocuteurs, création d'occurrences différentes, etc). Un univers est par conséquent cohérent.

Un discours donne donc naissance à une suite $\mathrm{U}_{0}$, $\mathrm{U}_{1}, \ldots, \mathrm{U}_{\mathrm{n}}$ d'univers. Un univers est pris par défaut, celui de l'énonciation courante, noté $\mathrm{U}_{0}$.

Le passage d'un univers à un autre s'accompagne d'un béritage d'objets de l'ancien vers le nouveau. De plus, dans chaque univers, i] y a héritage de l'intension vers l'extension (voir le processus dans l'extensionnel).

Une application ${ }^{\perp}$ possible de ce concept est la suivante : une interface pour un systeme expert d'aide à la conception de réseaux d'ordinateurs, le système présenté étant l'outil de représentation des connaissances. La partic intensionnelle d'un univers comprend les propriétés générales d'ui réseau et de ses composants. Par contre, un réseau particulier en cours de conception sera dans la partie extensionnelle de l'univers.

\subsubsection{Les notions et les types}

Il existe des objets pré-assertifs, c'est-ă-dire neutres par rapport à l'opposition nom/prédicat : "grand" par exemple, peut se comporter comme un prédicat ("être grand") ou comme un nom ("le grand"), suivant le réseatu de rclations qui lui est associéc. Ce sont les notions. Ces notions peuvent être atomiques ("rouge","rapide", ...) on construites ("chien noir mouille").

Les notions atomiques sont considérées comme des termes primitifs du système car elles ne sont associées à aucun domaine. Elles figurent comme des sous-objets indecomposables (terminaux) dans d'autres objets.

La notion construite, lorsqu'elle est munie d'une assertion (telle "le chien noir mouille") constitue ce que l'on appelle un type.

\subsubsection{Les univers intensionnels}

Ces univers fonctionnent comme des "réservoirs de savoir". Ils sont formés des types dont on a parlé plus haut. Des méta-types sont également définis pour décrirc des types d'objet dans l'univers intensionnel.

\subsubsection{Les univers extensionnels}

Un discours (texte, dialogue, ... ) introduit des univers extensionnels, liês aux conditions d'énonciation du texte. On peut décrire le processus de la façon suivante : un syntagme nominal du discours donne naissance à un nom 'N'. Ce nom est un objet du discours.

S'il correspond à un type déjà construit, on mettra en ceuvre un processus d' héritage des sous-objets du type au nom $N$. Ce nom portera d'autres informations : son statut correspondant aux conditions d'énonciation et des sous-objets qui lui sont propres et qui sont construits dans le discours.

Si le nom ne correspond à aucun type intensionnel de la base et que $\mathbf{N}$ désigne un nouveau type, un nouveau type est crée dans l'intensionnel. Si par contre, $\mathbf{N}$ désigne un objet particulier, on créera, dans l'extensionnel, un objet individuel nouveau.

\subsection{Le schéma d'un type}

On appellera schéma d'un type la description de ce type dans l'univers intensionnel. La description d'un type est constituée d'une liste de propriétés communes aux objets de ce type, et désignés par des noms d'attributs.

${ }^{1}$ Cette application est réalisée dans le projet Esprit $\mathrm{MMI}^{2}$ (Multi-Mode Interface for Maul-Machine Interaction). 
Les différents atıributs associés à un type sont :

- le statut: précise la nature de l'univers on l'on se situe. Il renvoie soit à "intension", soit d "extension",

- L'univers : indique le nom de l'univers courant $\left(\mathrm{U}_{0}, \mathrm{U}_{1}, \ldots, \mathrm{U}_{n}\right)$,

- La catégoric : indique la valeur IND si on a affaire à un individu, CL si c'est une classe.

La partie définitionnelle va contenir des informations de nature encyclopédique, considérées comme uil savoir permanent, ou des informations liées a un savoir construit datis l'interaction en cours (du dialogue par cxemple). Elle comprend les sous-objets suivants :

- Dénomination : ils indiquent le nom typique de l'objet.

- Autres-noms : comme son nom l'indique, cette catégoric d'attributs regroupe les noms synonymes.

- Netionuels ou Typiques: ils representent des notions atomiques ou des types, liés au type decrit.

- Structurels : ils decrivent les propriétés structurelles dı type.

- Fonctifs : ils indiquent une relation entre le type considéré et d'autres types. Cette relation est représentée en surface par un verbe ou une forme verbate nominalisée. Ce verbe peut représenter un état ou un processus. La scmantique des procès est décrite dans des objets de nature particulière. Dans le cas des processus, l'objet est un schéma d'action ${ }^{2}$ comportant une précondition, une post-condition, un résultat et un produit associés, ainsi que la méthode, qui est la description du processus lui-même. Comme indiqué dans l'introduction, nous ne nous intéressons pas ici aux schémas d'action [Gallo \& Rouault 92].

En résumé, le schéma d'un type est décrit de la façon suivante :

TYPE

Statut
Univers
Categorie
Dénomination
Autres-Noms
Notions
Types
Suructurels
Fonctifs

\section{3 - HORMALISATION DU MODELE}

\subsection{Les bases logiques}

La logique sous-jacente à notre système est une logique d'ordre 1, avec des termes fonctionnels d'ordre supérieur. la particularité de ces termes est leur constniction fondéc

\footnotetext{
${ }^{2}$ On peut comparer les schémas d'action à des méthodes au sens de la programmation objet.
}

sur deux primitives : l'uue ilotéce epsilon " $\varepsilon$ " issue du Calcul des Noms et la seconde est la relation d'ingrédience de la Méréologie [Miéville 84] [Lesniewski 89]. Dans cette section, nous ne pouvons aborder dans les détails les caractéristiques de chacune des théories en jeu. Nous nous bornons de tenter (la tâche est rude) d'expliquer ces deux foncteurs, chacun dans le cadre de sa theoric, uniquement daiss le sens où ils serviront de constructeurs principaux de notre modele objet.

Le Calcul des Noms, conme son nom l'indique, manipule des noms. Ces noms peuvent être individuels ou généraux. Les premiers dénotent un seul objet par opposition aux seconds, dont l'extcnsion a un clécment ou plus. Le foncteur epsilon va former une proposition dı type $A$ E b, qui se lit "A est-un b", où $A$ est un nom individuel et b un nom gênéral (qui peut être individuel : daus ce cas, on lit "est-le").

Par comvention d'ecriture, les majuscules désignent les noms individuels et les minuscules des noms généraux.

Exemple: Satune $\varepsilon$ plancte pour " $A \varepsilon$ b" Ravaillac e l'Assissin de Henti IV pour " $A \varepsilon B$ "

Il est interessant d'ajouter qu'unc algèbre des noms donne des résultats qui ont un correspondant en Theoric des Ensembles. On peut considérer l'epsilon comme mettant en relation un objet et une classe dite distributive.

La Méréologie introduit une signification nouvelle de la relation d'appartenance, prise comme partie/tout, telle qu'on la trouve par exemple dans "la roue est une partic de la bicyclette". Cette relation, notée "ingr" (pour ingrédience), va donner naissance à des ensembles appelées classes collectives. Cette classe méréologique est elle-même construite à partir d'une classe distributive par un foncteur Kl(a). Elle va contenir des éléments qui ne sont pas forećment de même nature comme dans la classe distributive.

Exemple : l'extension de la classe distributive planète contient neuf éléments (les neuf planètes) ct rien d'autre. Par contre, la classsc collective Kl(planete) va contenir, en plus de toutes les planètes, tous les ingrédients (ou parties) possibles des planetes : les anneaux de Saturnc, la tîche rouge de Jupiter, etc. Donc:

Tâche rouge de Jupiter $\varepsilon$ ingr( $\mathrm{Kl}($ planète) )

Après cet aperçu des foncteurs primitifs, nous allons voir leur utilisation dans le modèle.

\subsection{Caractéristiques du modèle}

L'originalité essentielle du modele est d'être fondée sur le nom. De plus, comme dans la plupart des systemes orientés objets, toutes les entites et les données d'une application sont des objets : un simple entier est autant un objet qu'une structure complexe.

Ainsi, tout concept, objet, personne est un objet identifié par un nom. Une entité du monde réel est désignée par un nom particulier d'objet qui lui est propre. On peut remarquer que les types sont des objets 
comportant an statut "intensionnel". Deux types sont réputés différents s'ils different par au moins un sousobjet. Un type est désigné par un nom individuel.

Afin de décrire les différents liens existant dans un univers, le schéma suivant reprend les différentes associations :

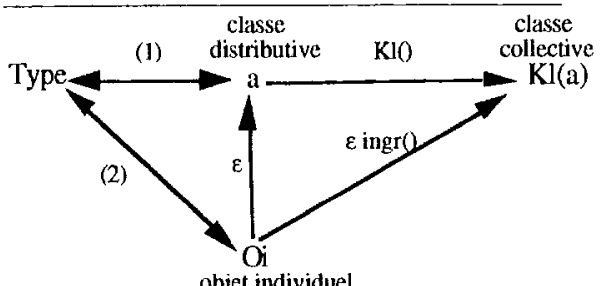

INTENSIONNEL

EXTENSIONNEL

Un objet particulier $O_{i}$ est considéré comme un élément de la classe distributive 'a' par la relation " $\mathrm{O}_{\mathbf{i}} \varepsilon$ a". Tous les objets vérifiant cette relation sont les éléments de la classe et font partie de ce que l'on appelle l'extension du nom ' $a$ '. On appelle ingrédients de $\mathrm{Kl}(\mathrm{a})$ les objets vérifiant " $\mathrm{O}_{\mathbf{i}} \varepsilon$ ingr(Kl(a))".

Tout nom général de classe distributive ou tout nom individuel d'objet de l'univers extensionnel peut être associé à un type (intensionnel). On considére qu'un type, nom individuel unique dans le système, décrit les propriétés caractéristiques.

Le passage entre Intensionnel et Extensionnel de l'univers peut être réalisé par divers foncteurs :

Les relations de (1) dans le schéma:

- Type-cl(classe $e_{i}$ qui associe un type aux éléments d'une classe distributive : "T $\varepsilon$ Type-cl(classe $\left.e_{j}\right)$ ".

- valeur-cl(Type $\mathrm{i}_{\mathbf{i}}$ associe une classe distributive à un type.

Les relations de (2) dans le schéma :

- Type-ind(Objet $\left.{ }_{i}\right)$ qui associe son type à un objet individuel tel que "T \& Type-ind(Objet $\left.{ }_{\mathrm{i}}\right)$ ".

- Valeur-ind $\left(\right.$ Type $_{\mathrm{i}}$ ) associe un nom d'objet individuel à un type.

Les objets de l'extensionnel d'un certain type auront les mêmes propriettés que celles du type associé.

\section{3 - Formalisation du schéma}

Chacune des propriétés du type sera considérée comme un ingrédient du type. Par conséquent, la structure de type contient des ingrédients, eux-mêmes étant des noms individuels d'objets, associés à des noms de type, qui à leur tour ont leurs propres ingrédients, etc. On a donc une structure d'objets complexes, le niveau le plus bas étant composé d'éléments dits "ingrédients atomiques", que l'on ne peut décomposer.
Ainsi, les noms de types (tels que entier, booléen, chaîne, réel, etc ) désignent des notions atomiques. Ceux-ci sont désignés comme faisant partie de l'extension du nom 'atome' défini en Méréologie : "J $\varepsilon$ atome".

On considère donc les noms d'attributs comme ingredients d'un type ; on associe à ces noms des types d'objet par un foncteur Type-de-valeurs().

Tout objet hérite de la structure de son type. Les ingrédients d'un objet sont ses sous-objets, et à chaque sous-objet est rattaché un attribut par le foncteur Nom_atto.

Nous donnons un exemple qui comprend la description du type universel (type de tous les types d'objets), d'un type particulier Personne et enfin une instance de Personne.

- Commençons par décrire le type universel T :

Statut $\varepsilon$ ingr $(T)$

Univers $E$ ingr( $(T)$

Catégorie $\varepsilon$ ingr $(T)$

Autres-noms $\varepsilon$ ingr $(T)$ $-$

Structurels $E$ ingr( $(T)$

Fonctifs $E$ ing(T)

Les uypes de valeurs:

Type-de-valeurs(Statut) $\varepsilon$ Valeur-statut

Type-de-valeurs(Univers) \& Valeur-univers

Type-de-valeurs(Structurels) \& Type-structurels

Type-de-valeurs(Fonctifs) $\varepsilon$ Type-schéma-action

Valeur-statut $\leq T$

Type-ind(INT) $\varepsilon$ Valeur-statut

Type-ind(EXI) $\varepsilon$ Valeur-statut

Valeur-univers $\leq T$

$$
-
$$

Type-structurels $\leq T$

Type-schéma-action $\leq \mathrm{T}$

- Soit le type Personne $\leq T$

Personne :

Statut $\varepsilon$ INT

Univers $\varepsilon$ Uo

Nom $\varepsilon$ ingr(Structurels)

Type-de-valeurs(Nom) $\varepsilon$ Liste-de-noms

Prénom $\varepsilon$ ing(Structurels)

Type-de-valeurs(Prénom) e Liste-de-noms

Age $\varepsilon$ ingr(Structurels)

Type-de-valeurs(Age) $\varepsilon$ Int-0-150

Date-naiss $\varepsilon$ ingr(Structurels)

Type-de-valeurs(Date-naiss) E Date Jour $\varepsilon$ ingr(Date-naiss)

Type-de-valeurs(Jour) $\varepsilon$ Int-1-31 
Mois $\varepsilon$ ingr(Date-naiss)

I'ype-de-valcurs(Mois) \& Int- I-12

All $\mathrm{E}$ ingr(Date-naiss)

Type-dic-valeurs $(A n)$ e Entior

- Soit une instance d'objet individuel Pierre Dupond de type Personne:

type-ind(Pierre Dupond) $\varepsilon$ Personne

Pierre Dupond :

Univers \& Uo

Statut \& EX'ı

Catégoric e IND

$\rightarrow$

Nom $\varepsilon$ Dupond

Prénume Picre

Age $\mathbb{E} 38$

Date-naiss :

Jour \& 25

Mois E. 5

All E 1953

\section{4 -ORGANISATYON DES CONNAISSANCLS}

La représentation d'un texte dans un univers extensionnel (voire dans plusicurs) se fait a l'aide des objets crés par ce texte. Par ailleurs, il y a dans l'univers extensionnel des objets provenant, par héritage, de l'univers intensionnel correspondant.

Ces objets ne sont pas isulés. En effet, la présence, dans un objet (intensionnel ou extensionnel) de sous-objets structurels et de sous-objets fonctifs met, de fait, cet objet en relation avec d'autres. Et ces liens entre objets crêent un réseau d'objets, appelé cotopie [Berrendonner \& Roualu 911. Bien évidemment, une cotopic se modifie à mesure que le discours se déroule.

Notre systeme tient compte de trois types de 'lien' dans cette cotopic :

\section{A - lien de classification}

C'est la hiérarchie crée par les liens entre types : on définit une relation de sous-typage qui vérifie les propriétés d'un treillis, oì par construction, chaque type hérite donc des proprictés de ses ancêtres.

Il existe un nom designant la classe distributive de tous les individus qui est noté "objet". Le type associé a la classe "objet" est noté T. Il représente le type universel de tous les individus extensionnels : type-cl(objet) E T.

Le foncteur de sous-typage est note ' $\leq$ '. C'est uue relation d'ordre particl.

Dire que le type ' $\mathrm{I}$ ' est un sous-type du type $\mathrm{T}$ ' implique que tout ingrédient de $\mathrm{T}$ 'est un ingrédient de $\mathrm{T}$ : $T \leq T^{\prime} \Rightarrow[\forall S]\left(S E \operatorname{ingr}\left(T^{\prime}\right) \Rightarrow S E\right.$ ingr(T) $)$
13 - lien de composition

C'est le licn entre un objet et ses sous-objets ingrédients. Un treillis móréologique découle également de cette construction structurelle.

\section{C. lien de référence}

C'est le lien existant entre deux types de nature indépendante. Lat référence est expriméce par l'attribut de catégoric "Fonctit". Ces liens fonment un résea entre les tyjus.

\section{5 - LES RAISONNEMENTS}

Le systène que nous décrivons a pour finalité de prendre elı compte, non seulentent les ratisomnements déductifs des systèmes logiques de Lesniewski, mais aussi des raisonnements inférentiels liés à des phénomènes des langues naturelles, comme l'anaphore. En conséquence, nous frisons coexister deux familles d'inférences. D'abord, les raisomements déductits portés pai le Cálcul des Nons et la Mérécologie : bien çue se situant daus des systènes particulicrs, ils iessortissent à la famille des raisomements logiques habituels et ne seront pas considertes ici.

L'une des originalités dı système réside evidemment dans la possibilite de memer des raisonnements inferrentiels dont la "conclusion" n'est que plausible. Ces raisonnements sont de plusieurs sortes suivant que l'on nodific le statut ou la partic definitionnelle d'un objet. Its sont a la lois inférentiels et déductifs, ce sont les raisonncinents par "abduction".

\section{1 - Inférences relatives à la partie "statut".}

Eu théoric, elles concenent le passage d'un univers à un autre, lc changement de catcgorie et les passages entre type, classe et individu. Nous ne considérons pas ici le premier cas. Lin ce qui concene les deux antres, nous avons les possibilités suivantes:

(1) Inference faisatut passer d'un type a un type ctominant (lans la hiérarchie des types (liyperonyme) :

"Le gendarne Labourbourax ... . Ce militaire ..." .

(2) Passige d'ull type à un sous-type : par ajout (do notions par exemple) ou modilication de proptiétes :

Appirition de "le chien noir mouille" alors que le type "chican" existe déjà.

(3) Passage d'un type à un individu extensionnel : "Je contais bien le GRILLON pare que j'ai eu l'opportunité de passer toute la nuit avec LUÍ dans mon sac de couchage".

(4) Passage d'un type à une classe exteusiomelle: "Quand la femme se rebiffe, LEUR mari essaye de s'éloigner pour ne pas répondre à leurs questions".

(5) Passage d'un individu extensionnel à un type:

"L'ANNEE 87 ne finit pas très hien. J'espère qu'ELLE commencera mieux en $88^{\prime \prime}$.

(6) Passage d'une classe extensionnelle à un type :

"Il y aura des APPAREILS qu'on placera dans l'oreille. Dans CET APPAREIL, on pourra mettre des bantes Inagnétiques". 


\section{2 - Inférences sur les dénominations}

Elles portent sur les sous-objets "Dénomination" et "Autres-noms", donc contiennent un passage d'une dénomination à une autre :

"Il y avait PLUSIEURS PETITES MAISONS, Je tne demandais à quoi ILS pouvaient bien servir" (ils = les préfabriqués).

\section{3 - Inférences sur les structurels}

Elles concernent le foncteur d'ingrédience :

(1) passage d'un sous-objet propriété à l'objet, représentant un individu ou une classe :

"Mon père vit DES POINTS NOIRS. Il se dépêcha de couper LES MORILLES avec sol opinel".

(2) "Nous vîmes apparaître des têtes casquées. Les C.R.S ..."

(3) "Sans même attendre qu'il soit mort, les Tunisiens renversent les STATUES DE BOURGUIBA. C'est vache pour le CHEVAL. Il n'avait rien fait fait de mal, le cheval" (il s'agit d'une statue equestre).

\section{4 - Inférences faisant intervenir les fonctifs}

Ici en l'absence d'un inventaire méthodique, nous nous contenterons de signaler les cas suivants d'inférences :

(1) Passage d'un objet à un autre objet :

"On protège laccès aux routeurs (OBJET 1) par mot de passe (OBJET 2)".

Le fonctif est "protéger-par(objet1, objet2)".

(2) Passage de l'action à un argument :

"IL NEIGE et ELLE tient".

\section{5 - Mise en oeuvre des raisonnements}

Chacun des modes de raisonnement précédents permet le passage d'un objet I à un objet I' ; autrement dit, partant de l'objet I, on infere un nouvel objet I', qui est ajouté à l'univers courant. Les sous-objets de cet objet sont alors disponibles pour de nouvelles inférences.

C'est ce que nous regroupons sous le terme genérique de raisonnement par abduction. Un tel raisonnement est donc formé d'une partie inférentielle puis d'une partie déductive ; il est représenté par une règle.

Il nous reste à préciser comment on met en auvre ces tègles.

L'application d'une règle est un appariement entre une cotopie et une séquence en langue naturelle. La cotopie est formée des objets déjà crés dans le discours, directement ou par des inférences. La séquence en langue naturelle forme un syntagme nominal et correspond à une partie nouvelle du discours à représenter. Elle est transformée en un couple formé d'une détermination, liée au statut des objets et d'une description ; ta description correspond à la partie définitionnelle des objets. Elle est supposée mise (par le système d'analyse automatique du français) sous la forme d'une composition de notions, par ((c(états) + africains) + francophones) + démocratiques)
Une telle description est donc une composition de descripteurs :

$(\ldots(((d 1)+d 2)+d 3) \ldots)$

Le problème de l'application d'une règle est donc celui de l'appariement d'une telle description avec les objets de la cotopie. Nous distinguons plusieurs cas :

(1) Appariement complet et direct entre la description et un objet de la cotopie c'est le cas de reprise directe comme dans "Et puis, il m'a remis sa carte. Et CETTE CARTE la voici"

(2) Appariement complet indirect : pour que l'appariement soit complet il faut que l'on opere des inférences sur l'objet ou sur la description : les inférences possibles ont été recensées ci-dessus.

(3) Appariement incomplet direct : une partie seulement de la description s'apparie avec un objet de la cotopie.

(4) Appariement incomplet et indirect : l'appariement indirect nécessite des inférences sur l'objet ou sur la description, ou sur les deux.

(5) Non-appariement.

Les conclusions à tirer d'un appariement incomplet ou d'un non appariement dépendent en particulier de l'application visée. Dans un dialogue homme-machine avec un système expert par exemple, l'appariement partiel peut conduire à une demande d'explication du système en direction de l'utilisateur, alors que le nonappariement devrait constater l'échec de la requête de l'utilisateur. Par contre, dans un dialogue d'extraction de connaissances, le non-appariement conduit logiquement à la création d'un nouveau type.

\section{6 - IMPLEMENTATION}

Une implémentation du système sous la forme d'instructions de la machine abstraite de Warren est en cours de conception.

On représente l'architecture de l'implémentation dans le schéma suivant :

\begin{tabular}{|c|}
\hline $\begin{array}{c}\text { Modile } \\
\text { objets }\end{array}$ \\
\hline ingr-ternes \\
E-termes \\
formules logiques du $1^{\circ}$ ordre \\
\hline Machine de Warren \\
étendue \\
avec mécanismes d'unification \\
sur les treillis
\end{tabular}

- Le premier niveau consiste en la machine abstraite de Warren étendue avec des mécanismes pour supporter 
l'appariement des objets.

Ce que l'on a appelé l'appariement va reposer sur l'opération d'unification sur les treillis de types et d'ingrédicnce. En effet, on va distinguer deux sortes d'unification du mécanisme d'inférence. La sémantique de chaque unification est attachce a la connaissance organisce dans chacun des treillis :

- dans le treillis des types, nous allons procéder à une intersection de types (qui correspond au plus grand des minorants) [Aït-Kaci \& Nasr 86].

- dans le treillis d'ingrédience, c'est une gênéralisation (1e plus petit des majorants).

- Le second niveau implémente le formalisme logique composé des formules du $1^{\circ}$ ordre, des $\varepsilon$-termes et des ingr-termes.

Cette solution semble plus avantageuse à côté d'une implémentation lisp ou prolog ctendu.

- Le troisième niveau lui implémente le modèle objet à l'aide du formalisme logique sous-jacent.

\section{7 - CONCLUSION}

Dans ce document, nous avons présenté un système inférentiel orienté objets pouvant ĉtre utilisé dans diverses applications en langues naturelles.

Nous avons insisté sur le formalisme de représentation de connaissance qui est à la base du raisonnement "naturel" que nous souhaitons réaliseri. Les différents types de raisonnement ont été illustrés par des exemples.

Le système présenté ici est en cours de construction. Les bases theoriques (linguistiques et logiques) sont définies. Le passage de la surface linguistique à la représentation comporte des zones résistantes, dont l'une des plus importantes est la distinclion intension/extension. Son repérage passe par une étude assez fine des operations d'operations.

\section{BHBLIOGRAPHIL}

[Allen 88] J. Allen, "Natural Language Understanding", Benj/Cummings Publishing, 1988.

|Ait-Kaci \& Nissr 86] H. Ait-Kaci et R. Nasr, "Login : A logic programming language with built-in inheritance", J. of Logic Programming, vol.3 n 3 , Oct. 1986

[Berrendonner 89] A. Berrendomer, "Sur l'inférence", daus Modeles du discours, Rubattel, P. Lang, Berne 1989

[Berrendonncr \& Rouault 91] A. Berrendonner et J. Rouault, "Sémantique des objets et Calcul des Noms", KMET 91, Nice, Avril 1991

[Brachmanl \& Levesque 85] R.J. Brachman et H.J. Levesque, "Readings in Knowledge Kepresentation", Morgan Kaufmann Publishers, 1985.

[Brachman \& Levesque 91] R.J. Brachman et H.J. Levesque, Artificial Intelligence, vol. 49, $\mathrm{n}^{\circ}$ 1-3. Special volume on Knowledge Representation, 1991.

[Fredj 92] M. Fred], "LESLOG : un systèmc intércuticl orienté objet fondé sur les systèmes logiques de Lesnicwski "Rapport de recherche CRISS, 1992 (a paraître)

[Gallo \& Rouault 92] M.C. Gallo et J.Rouault, "Les schemas d'action", Notes de recherche CRISS, 1992 (à parâtre)

[Lesniewski 89] St. Lesniewski, "Sur les fondements de la mathematique", traduit du polonais par Kalinowski, Hermès, Paris, 1989.

[Miéville 84] D. Mieville, "Un développement des systèmes logiques de S.Lesniewski", P. Lang, Benle, 1984.

[Richard $(0)] J .14$. Richard, "Les activités mentales: comprendre, raisonner, trouver des solutions", Armand Colin, Paris, 1990).

[Thayse 89] A. Thayse \& co, "Approche logizue de l'Inelligence Artificielle", vol. I \& 2, Dunod Infornatique, 1989.

[Zdonik 84] S. B. Zdonik, "Object Management System Concepts", Proc. of the $2 d$ ACM SIGOA conference on office information system.s, Toronto June 1984 / SIGOA Newsletters, vol. $5 n^{\circ} 21984$. 